\title{
Vertebral artery stenosis with subcortical ischemic infarcts and cortical atrophy without dementia presenting with TIA - case-report of a causality challenge
}

\begin{abstract}
Rationale: Subcortical infarcts with TIA, multi-infarct dementias and vertebral artery stenosis are well-defined entities. Uniqueness of this case-report stems from the combination of vertebral artery narrowing, multiple subcortical infarcts and cortical atrophy without dementia, presenting with facial weakness. Elucidating the causality was the challenge in this case.

Presenting concerns: A 45-year-old male executive complained of feeling unreal and difficulty in focusing while in office. Examination revealed normal cognition and a slight drooping of left lower face.

Investigations and diagnoses: Brain MRI imaging revealed significant diffuse cortical atrophy, prominent lateral ventricles, multiple bilateral subcortical $3-4 \mathrm{~mm}$ infarcts, periventricular ischemia and significant narrowing of right vertebral artery. A diagnosis of transient ischemic attack (TIA) was made.
\end{abstract}

Intervention and outcome: The patient was admitted in ICU and put on statin and aspirin. Within 24 hours facial droop disappeared. One-week follow up revealed no neurological and cognitive deficit. Patient was advised to continue medication and modify his diet and lifestyle.

Keywords: subcortical infarct, cortical atrophy, stroke, vertebral artery stenosis, double vertebral artery
Volume 9 Issue I - 2019

\author{
Sanjoy Sanyal,' Thomas Thomas, ${ }^{2}$ Debjyoti \\ Talukdar ${ }^{3}$ \\ 'Professor and Department Chair, Neuroscientist and Surgeon, \\ All Saints University College of Medicine, West Indies \\ ${ }^{2}$ Assistant Professor, Department of Preventive Medicine, All \\ Saints University College of Medicine, West Indies \\ ${ }^{3}$ Research Student, All Saints University College of Medicine, \\ West Indies
}

Correspondence: Sanjoy Sanyal, 1028 Austin Pond Drive, Cary, North Carolina, NC 27519, USA, Tel + I 784593 4388; Email sanyal.sanjoy8@gmail.com

Received: October 23, 2018 | Published: January 28, 2019

\section{Introduction}

Subcortical infarcts with transient ischemic attacks (TIA), multiinfarct (vascular) cortical or sub-cortical dementia, cortical atrophy and vertebral artery stenosis are well-defined entities. Uniqueness of this case-report stems from a combination of unilateral vertebral artery narrowing, double bifid vertebral artery on one side, multiple bilateral subcortical infarcts and diffuse cortical atrophy but without dementia, presenting with supra-nuclear facial weakness. Elucidating the cause-effect relationship was the diagnostic conundrum.

\section{History and presenting concerns}

While in office one day during a meeting the patient suddenly complained of feeling dizzy and unreal. He said he had difficulty in focussing on the conversation or on anything, for that matter. This was his first such episode. The patient was a 45 -year old unmarried male Filipino executive. He was a non-smoker, though he did consume alcohol occasionally on social functions and at home. He had no known history suggestive of diabetes mellitus, hypertension or cardiac ailments. Being an office executive, his lifestyle was completely sedentary, both in and out of office, and he did not participate in any form of physical workout. He consumed only non-vegetarian diet. There was no significant family history. He suffered from attacks of claustrophobia. He had a fear of sitting next to big people and he was morbidly scared of crossing any vehicular street unless accompanied by another person and led by the hand.

\section{Physical examination}

Examination revealed a short-statured slightly built young middleaged male of distinct oriental extraction. He was alert, conscious, had normal cognition, and responded cogently to all commands. However, there was a slight drooping of his left lower face and left angle of mouth, with flattening of left naso-labial furrow. Pulse and blood pressure were normal. There were no other significant findings, neurologically or systemically.

\section{Investigations and imaging findings}

Labs showed high triglycerides and cholesterol. Brain computerized tomogram (CT) scan revealed diffuse cortical atrophy disproportionate for age and prominent lateral ventricles. Extracranial Color Doppler demonstrated laminar flow in major cervical and cranial vessels.

T2 Magnetic Resonance Imaging (MRI) of brain showed multiple bilateral old subcortical lacunar (3-4mm) hyperintense infarcts, more on left side, generalized cortical atrophy disproportionate for age and prominent lateral ventricles in Fast Gradient Recalled Echo Serial (FGRE-S) axial sections (Figure 1). FGRE-S coronal and parasagittal sections also showed same findings as well as hyperintense periventricular ischemia (Figure 2). 


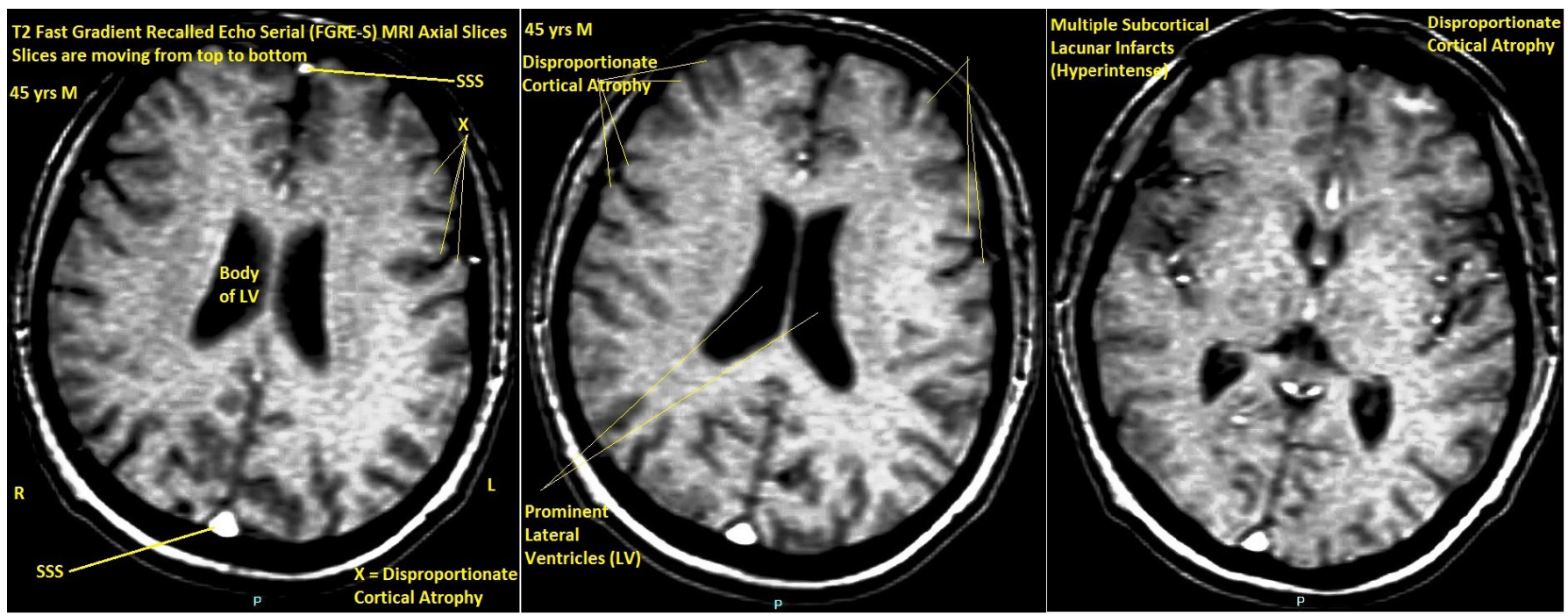

Figure I T2 Magnetic Resonance Imaging (MRI) of brain showed multiple bilateral old subcortical lacunar (3-4mm) hyperintense infarcts, more on left side, generalized cortical atrophy disproportionate for age and prominent lateral ventricles in Fast Gradient Recalled Echo Serial (FGRE-S) axial sections

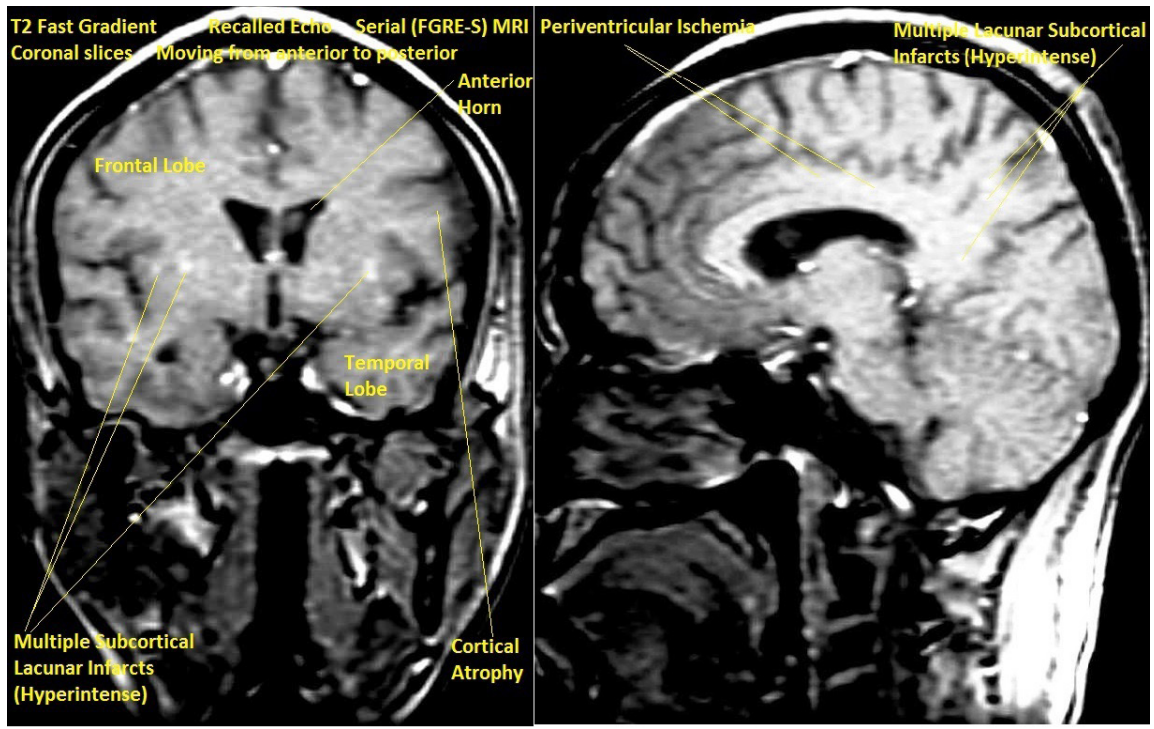

Figure 2 FGRE-S coronal and parasagittal sections also showed same findings as in Figure I, as well as hyperintense peri-ventricular ischemia

3-D Time of Flight Spoiled Gradient Recall (TOF SPGR) Echo Acquisition Magnetic Resonance Angiograms (MRA) of VertebroBasilar and Carotid Arteries revealed significantly narrow vertebral artery in a composite 3-D view (Figure 3). Sequential films in 3-D TOF SPGR mode with the head rotating in a clockwise direction revealed narrow bifid right vertebral artery, accessory right vertebral artery, abnormal communication of right vertebral artery with right internal carotid artery and normal left vertebral and internal carotids (Figures 4-6).

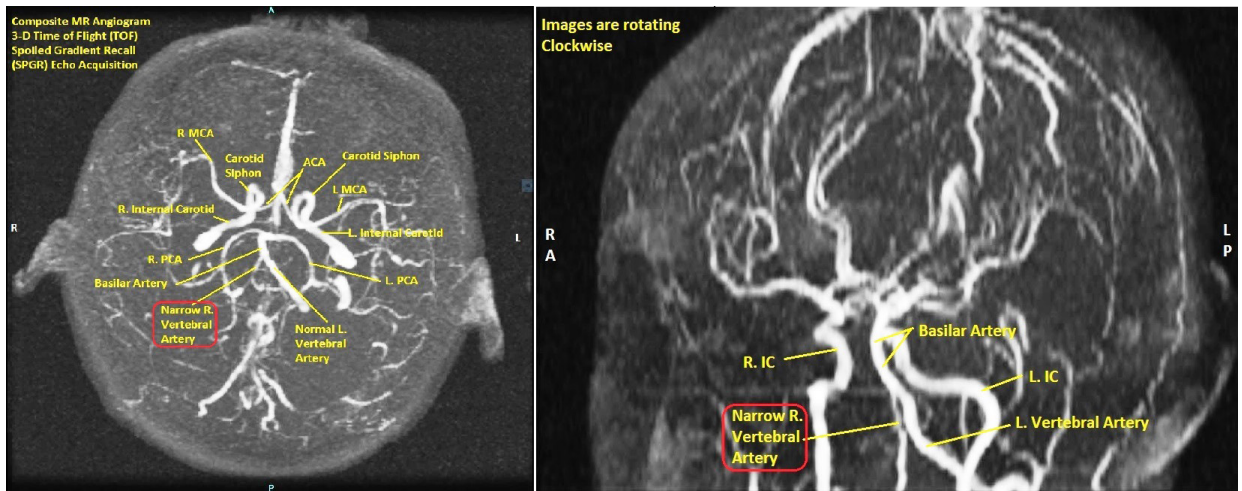

Figure 3 3-D Time of Flight Spoiled Gradient Recall (TOF SPGR) Echo Acquisition Magnetic Resonance Angiograms (MRA) of Vertebro-Basilar and Carotid Arteries revealed significantly narrow vertebral artery in a composite 3-D view

Citation: Sanyal S,Thomas T,Talukdar D.Vertebral artery stenosis with subcortical ischemic infarcts and cortical atrophy without dementia presenting with TIA - case-report of a causality challenge.J Neurol Stroke. 2019;9(I):I8-24. DOI: 10.15406/jnsk.2019.09.00340 

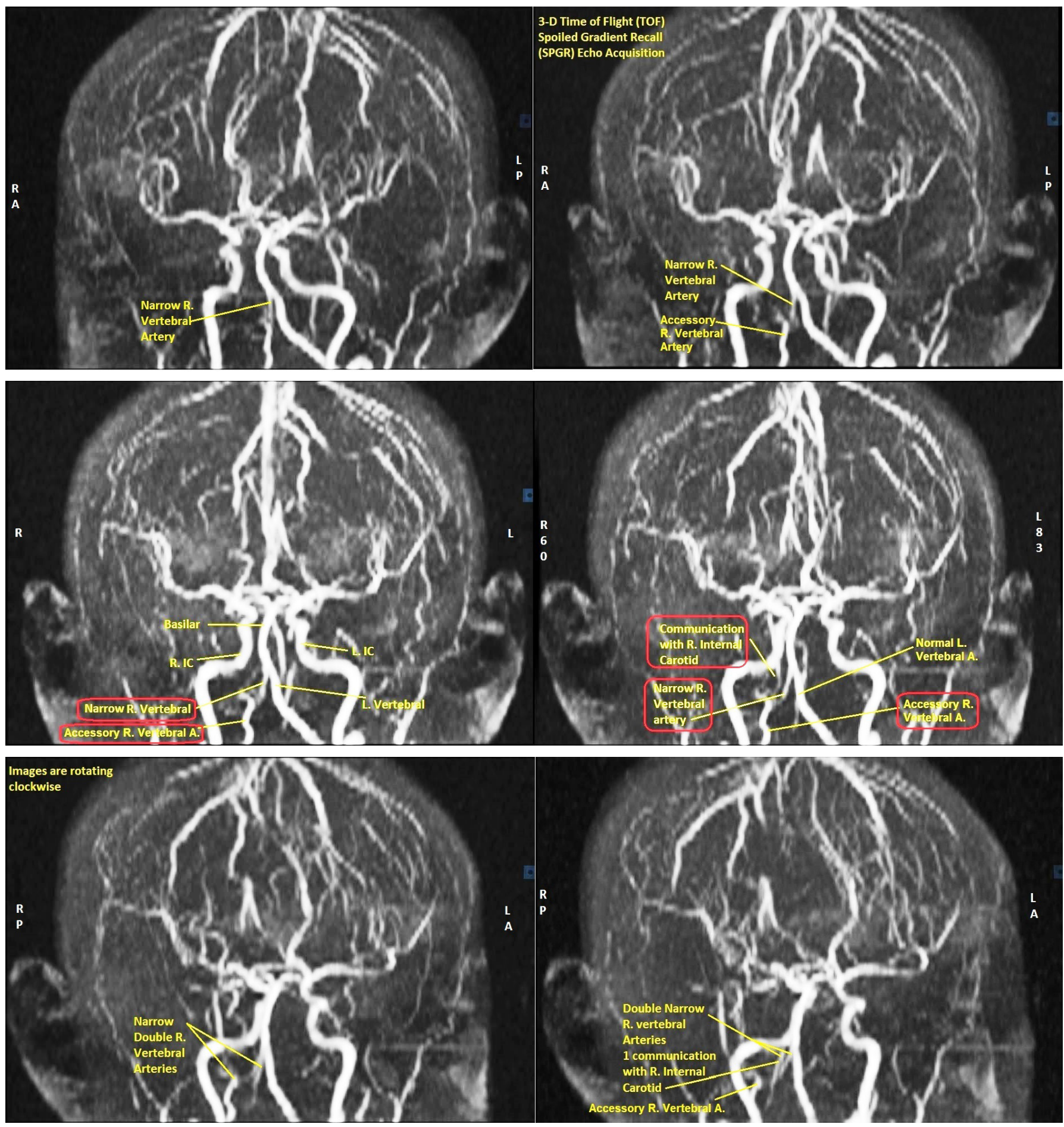

Figures 4 to 6 Sequential films in 3-D TOF SPGR mode with the head rotating in a clockwise direction revealed narrow bifid right vertebral artery, accessory right vertebral artery, abnormal communication of right vertebral artery with right internal carotid artery and normal left vertebral and internal carotids

2-D TOF SPGR axial MRA sequence going from cervical region up to base of pons also revealed narrow, bifid right vertebral artery, accessory right vertebral artery, normal left vertebral and bilaterally normal internal carotid arteries (Figure 7 \& 8), essentially corroborating the findings of the 3-D TOF SPGR MRA sequence.

\section{Diagnostic focus and assessment}

A clinical diagnosis of fresh transient ischemic attack (TIA) affecting the right cerebral hemisphere, with left supra-nuclear facial palsy was made. Correlating all the imaging findings with the present diagnosis and elucidating the cause-effect relationship (causality) was the diagnostic challenge.

\section{Therapeutic focus and assessment}

The patient was admitted in Intensive Care Unit (ICU) under Neurological care. He was administered Simvastatin $40 \mathrm{mg}$ O.D. and Aspirin $81 \mathrm{mg}$ O.D. He was prescribed low-calorie, low-salt diet. After an uneventful night, next morning his facial droop had noticeably 
decreased. He was feeling much better already. However, he was kept under observation for the remainder of 24 hours. He was discharged on the same medications and dietary advice. He was further advised to be on bed rest for the next 5 days and continue the medications.
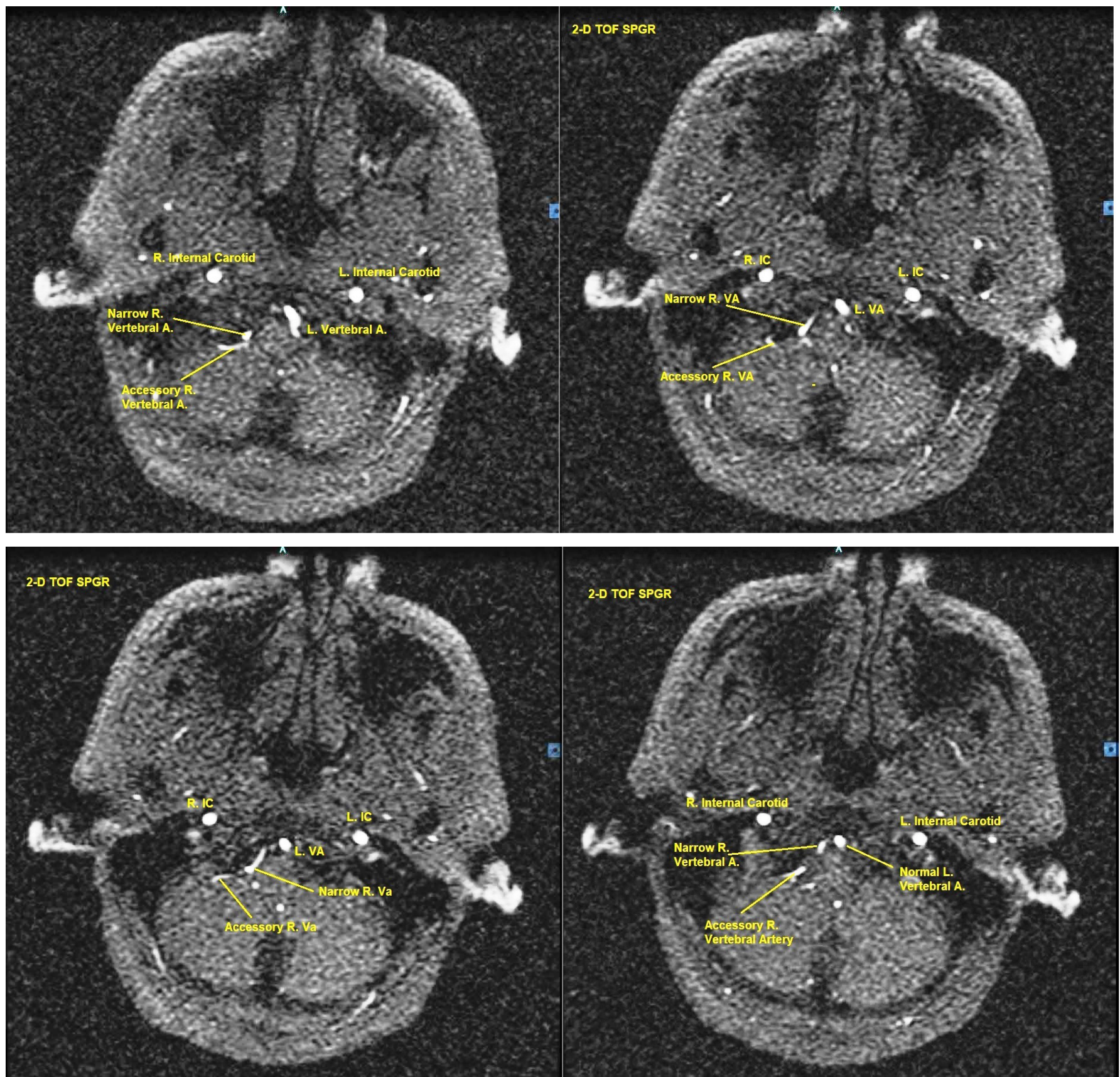

Figures 7 and 8 2-D TOF SPGR axial MRA sequence going from cervical region up to base of pons also revealed narrow right vertebral artery, accessory right vertebral artery, and normal left vertebral and bilaterally normal internal carotid arteries, essentially corroborating the findings of 3-DTOF SPGR MRA sequence

\section{Follow-up and outcomes}

One-week follow up revealed no neurological and cognitive deficit. His left lower facial droop had disappeared completely. He was speaking and thinking normally. He had started working in his office as usual. Patient was advised to continue medication and modify his diet and lifestyle.

\section{Patient perspective}

Once the diagnosis and outcome was explained to the patient, being an erudite and intelligent individual, he accepted it without qualms, expressed his relief that the present crisis was defused, conveyed his gratefulness to his colleagues who helped him and the healthcare providers who participated in his care and promised to take all the precautions advised to him and do the needful to prevent a future recurrence of similar, or worse episode.

\section{Timeline}

The temporal sequence of patient's history, progress and follow-up is shown in Figure 9.

Citation: Sanyal S, Thomas T, Talukdar D.Vertebral artery stenosis with subcortical ischemic infarcts and cortical atrophy without dementia presenting with TIA - case-report of a causality challenge. J Neurol Stroke. 2019;9(I): I8-24. DOI: I0.15406/jnsk.2019.09.00340 


\section{Timeline of Patient's Case History}

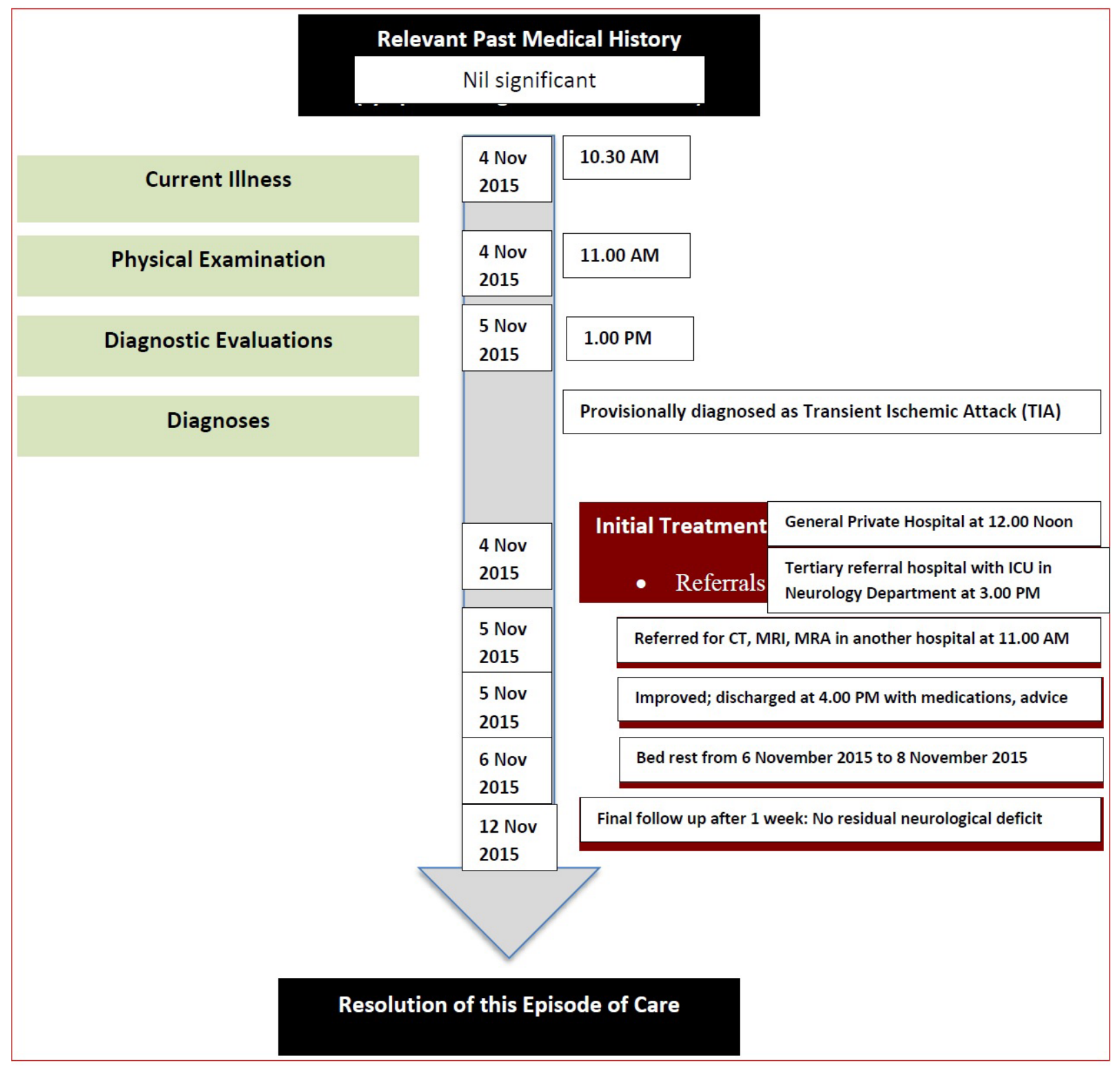

Figure 9 Temporal sequence of patient's history, progress and follow-up (case management) as described in the case-report

\section{Discussion}

\section{Transient ischemic attack and stroke}

The old definition of transient ischemic attack (TIA) was clinical syndromes of focal brain ischemia lasting less than 24 hours. However, most so-called TIAs more than 1 hour were found to have some degree of brain infarction on imaging. Therefore the current definition is transient clinical symptoms of stroke without evidence of fresh infarction on brain imaging. ${ }^{1}$ On the basis of this definition the left-sided upper motor neuron facial palsy may be considered as a manifestation of TIA.

TIA is basically a harbinger of a full-blown stroke and has the same origin and etiopathogenesis. While in a full-blown ischemic stroke, a blood clot blocks an artery to the brain, in a TIA the blockage is brief, at least for the time being, and there is no permanent brain damage, as assessed by imaging. ${ }^{1-3}$

In this case the TIA in the form of upper motor neuron (UMN) leftsided facial drooping was most probably due to cerebral subcortical small vessel disease (SVD), possibly in the region of corona radiata, around the lateral ventricles or in the internal capsule. The presence of numerous old lacunar (3-4mm) subcortical infarcts and periventricular ischemia would lend credence to this possibility. ${ }^{1}$ The vertebral arterial abnormalities were most probably incidental findings insofar as the UMN facial weakness was concerned. Even a single episode of TIA should be investigated thoroughly. Untreated, 1 in 3 cases of TIA have frank cerebro-vascular accident (CVA) within 5 years and $20-35 \%$ cases have permanent cerebral infarction. ${ }^{1,2}$ 


\section{Multi-infarct cortical atrophy and dementia}

This case had multiple bilateral lacunar $(3-4 \mathrm{~mm})$ subcortical infarcts, more on the right side, and periventricular ischemia, all of which were old. The diffuse bilateral cortical atrophy may be attributable to the numerous old lacunar infarcts, while the ventricular prominence may be explained as a compensatory mechanism to the cortical atrophy on the principle of hydrocephalus ex-vacuo. ${ }^{1}$

Notably, this case had no features of dementia. While multiinfarct (vascular) dementia is generally considered as the secondmost common cause of dementia worldwide, not all cases of multiple infarcts or cortical atrophy have dementia. ${ }^{4} \mathrm{He}$ was claustrophobic, had a fear of sitting next to big people and morbidly scared of crossing any street unless accompanied by another person and led by the hand. Psycho-neurotic features were most probably incidental findings, unrelated to either vertebral artery stenosis, cortical atrophy or multiple bilateral subcortical infarcts.

\section{Vertebral artery abnormalities}

This case had a narrow bifid right vertebral artery and a duplicate ('accessory') right vertebral artery, but there were no symptoms solely attributable to the vertebro-basilar circulation. Vertebral artery stenosis is well documented in the literature. ${ }^{5-11}$ Most are descriptions of symptomatic stenosis with vertebro-basilar posterior cranial fossa manifestations. ${ }^{5-7} 20 \%$ of strokes are caused by vertebral artery stenosis. ${ }^{8}$ The prevalence of vertebral artery stenosis without vertebro-basilar symptoms, as in this case, is poorly quantified, though studies put it at approximately $8 \% .{ }^{9,10}$ Such patients have a higher risk of developing posterior circulation ischemic stroke than those without stenosis. ${ }^{10}$ Therefore it is more important than ever for this group of patients to maintain aggressive modification of lifestyle and conservative measures.

Besides being stenosed and hypoplastic, the right vertebral artery was also bifid, as seen in 3-D Time of Flight (TOF) Magnetic Resonance Angiography (MRA). Duplication of vertebral artery is extremely rare, the incidence being less than $0.3 \% .^{11}$ Several embryonic mechanisms have been postulated, all of them directly or indirectly attributable to failure of regression of embryonic cervical intersegmental arteries. They are asymptomatic in most cases and do not carry any special clinical significance except insofar that they may constitute a diagnostic conundrum or may be encountered unexpectedly during vascular intervention procedures. ${ }^{11}$

Apart from narrow bifid right vertebral artery, this case also had an 'accessory' right vertebral artery, with communication with the right internal carotid artery as seen in 3-D TOF MRA images. This begs consideration of the possibility of a Persistent Primitive Artery (PPA). ${ }^{12}$ Primitive Arteries (PA) are embryonic communications between the internal carotid and vertebra-basilar systems before the development of posterior communicating (PCOM) artery. Going from superior to inferior the PA's are trigeminal, otic, hypoglossal and pro-atlantal arteries..$^{13}$ After development of the PCOM, the PA's disappear early in embryonic life. Rarely one may persist after birth; then it is referred to as Persistent Primitive Artery (PPA). PPA's are very rare occurrences, ranging from $0.1 \%$ to $0.6 \%$ of cerebral angiograms. They are usually unilateral. PPA is often associated with hypoplastic vertebral artery. ${ }^{14}$ Judging by the location of the right 'accessory' artery at the lower end of the vertebral artery in this case and its communication with the right internal carotid, and the fact that the right vertebral artery was narrow, it may be postulated that this case had a right persistent hypoglossal artery (PHA). Such PPA's, albeit rare, may be source of aneurysms and may be the cause of strokes, especially if mishandled during vascular interventional procedures. ${ }^{13,15}$

The vertebral artery anomalies in this case was diagnosed on the basis of 2-D and 3-D Time of Flight (TOF) Spoiled Gradient Recall (SPGR) Echo Acquisition Magnetic Resonance Angiography (MRA), which is a non-invasive procedure. ${ }^{16}$ The choice of imaging techniques ranges between Intra-arterial Angiography (IAA), Duplex Ultrasound (DUS), Computerized Tomographic Angiography (CTA) and Contrast-Enhanced Magnetic Resonance Angiography (CE-MRA) ${ }^{8}$ The first is an invasive procedure but it is considered as the international reference standard. The others are non-invasive procedures. An important consideration is to decide on the best imaging technique to determine cerebral vascular abnormalities, if any, symptomatic or otherwise. While data in this regard is limited, the current consensus of opinion tends to tilt in favor of CE-MRA, followed by CTA and lastly by DUS. ${ }^{8}$

\section{Study characteristics}

The obvious strength of this case report stems from the fact that latest non-invasive MRI and MRA imaging techniques were employed to diagnose the case, and the unique combination of imaging findings that appeared unrelated to the present episode, yet they were significant findings. ${ }^{8,16}$ The other significant point was that though there was definite bilateral cortical atrophy, with compensatory ventricular prominence, and multiple bilateral sub-cortical infarcts and periventricular ischemia, albeit old, there was no clinical evidence of cognitive deficit or dementia in this patient. ${ }^{4}$

The present episode of TIA was superimposed on the pre-existing old findings, and may lead one to make a wrong diagnosis if not assessed carefully. Secondly, cases with PPA have a higher risk of strokes and aneurysms. ${ }^{13,15}$ These should be the carry home messages from this study. The obvious limitation of this study was that this is a single case-report; therefore it cannot be generalized to a wider population base. Therefore the next focus should be collect a larger series of similar cases and try to establish a predictive model of TIA based on the collective findings.

\section{Conclusion}

The TIA was most probably due to cerebral small vessel disease rather than vertebral artery stenosis, which was an incidental finding. Even a single episode of TIA should be investigated thoroughly. Untreated, one-third cases have frank stroke within 5 years. While the ventricular prominence may be attributable to cortical atrophy, not all cases of multiple infarcts or cortical atrophy have dementia. Psychoneurotic features in this case may also be incidental findings. With one established episode of TIA and the presence PHA, aggressive medical management and close follow up is essential to prevent appearance of full-blown stroke in either or both cerebral circulations.

\section{Informed consent}

The patient donated all his imaging data on a Digital Imaging and Communications in Medicine (DICOM) compact disc (CD) to the principle author and explicitly gave consent for all his medical and imaging data to be used for the purpose of medical discussion, presentation or publication by the medical community.

\section{Acknowledgments}

The cooperation and contribution of the patient himself towards the publication of this material is gratefully acknowledged. 


\section{Conflicts of interest}

The author declares no conflicts of interest.

\section{References}

1. Lawrence K Loo. Kochar's Clinical Medicine for Students. $6^{\text {th }}$ ed. Chapters 107 to 117 ,pp. $652-693$

2. Mayo Clinic. Diseases and Conditions - Transient Ischemic Attack Symptoms and Causes.

3. Medical College of Wisconsin, Department of Neurology. Stroke, Neurointervention, and Critical Care. Copyright (C) 2018 MCW.

4. Kumar V, Abbas AK, Fausto N. Robbins and Cotran Pathologic Basis of Disease. $7^{\text {th }}$ ed. Elsevier Saunders; 2005.

5. De Cocker L, Compter A, Kappelle LJ, et al. Cerebellar cortical infarct cavities and vertebral artery disease. Neuroradiology. 2016;58(9):853857.

6. Compter A, Kappelle LJ, Algra A, et al. Nonfocal Symptoms are More Frequent in Patients with Vertebral Artery than Carotid Artery Stenosis. Cerebrovasc Dis. 2013;35(4):378-384.

7. Compter A, Van der Hoeven EJRJ, Van der Worp HB, et al. Vertebral artery stenosis in the Basilar Artery International Cooperation Study (BASICS): prevalence and outcome. J Neurol. 2014;262(2):410-417.
8. Khan S, Cloud GC, Kerry S, et al. Imaging of vertebral artery stenosis: a systematic review. J Neurol Neurosurg Psychiatry. 2007;78(11):12181225

9. Koch S, Bustillo AJ, Campo B, et al. Prevalence of vertebral artery origin stenosis and occlusion in outpatient extracranial ultrasonography. $J$ Vasc Interv Neurol. 2014;7(2):29-33.

10. Compter A, Van der Worp HB, Algra A, et al. Prevalence and Prognosis of Asymptomatic Vertebral Artery Origin Stenosis in Patients With Clinically Manifest Arterial Disease. Stroke. 2011;42(10):2795-2800.

11. Kim MS. Duplicated Vertebral Artery: Literature Review and Clinical Significance. J Korean Neurosurg Soc. 2018;61(1):28-34.

12. Weerakkody Y. Primitive arteries in the brain (mnemonic). Radiopedia.

13. Coulier B. Persistent Hypoglossal Artery. Journal of the Belgian Society of Radiology. 2018;102(1):28.

14. Alcalá-Cerra G, Tubbs RS, Niño-Hernández LM. Anatomical features and clinical relevance of a persistent trigeminal artery. Surg Neurol Int. 2012;3:111.

15. Srinivas MR, Vedaraju KS, Manjappa BH, et al. Persistent Primitive Hypoglossal Artery (PPHA) - A Rare Anomaly with Literature Review. $J$ Clin Diagn Res. 2016;10(1):TD13-TD14.

16. Kurvers AJ, Compter A, Wardlaw J, et al. Non-invasive imaging to detect vertebral artery stenosis. Cohrane Database of Systematic Reviews. In book: Cochrane Database of Systematic Reviews. Cochrane. 2014. 